\title{
Application of two-stage ADALINE for estimation of synchrophasor
}

\author{
Cheng-I Chen ${ }^{\mathrm{a}}$, Yeong-Chin Chen ${ }^{\mathrm{b}}$, Chao-Nan Chen ${ }^{\mathrm{b}}$, Chien-Kai Lan ${ }^{\mathrm{b}}$ \\ ${ }^{a}$ National Central University, No. 300, Jhongda Rd., Jhongli City, Taoyuan County 32001, Taiwan \\ ${ }^{b}$ Asia University, No. 500, Lioufeng Rd., Wufeng, Taichung 41354, Taiwan
}

\begin{abstract}
With the development of smart grid, the accurate estimation of phasor measurement is increasingly significant to help achieve the reliable transmission and distribution of power system. However, the power system frequency may deviate from its nominal value and lead to estimation errors for the most traditional approaches. To perform the accurate synchrophasor measurement, a technique based on adaptive linear neural network (ADALINE) is applied. With the high frequency resolution of autoregressive model, the variation of power system frequency can be effectively extracted. Through the testing results corresponding to different power quality disturbances, the total vector errors of several uncertainty examinations for synchrophasor measurement in IEEE Std. C37.118.1-2011 can be maintained in the permissible range.
\end{abstract}

Keywords: Adaptive linear neural network, synchrophasor, autoregressive model, total vector error, power quality disturbance

\section{Introduction}

The application of synchrophasors for the wide-area monitoring is one of important techniques in the protection of power systems. In recent years, the estimation of synchrophasors has suffered from interferences of power quality disturbances due to the widespread use of renewable generations and development of microgrids. The power system frequency deviation, harmonics/interharmonics, power quality events would lead to inaccuracy in the estimation of synchrophasors with the conventional fast Fourier transform (FFT) based methods [1]-[3]. In this paper, a technique based on the adaptive linear neural network (ADALINE) is applied to deal with this problem.

The ADALINE is an adaptive filter, which analyzes the power signals with the Fourier series. The advantages of ADALINE over the conventional FFT are the computational efficiency with the recursive mechanism and the capability to track the dynamic trends of power parameters [4]-[6]. However, the estimation of conventional ADALINE would be deteriorated when the power system frequency deviation and interharmonics are present [7]. This is because the decomposition model of ADALINE is still dependent on the Fourier analysis bases. From this phenomenon, it is revealed that the estimation of frequency is the most important task to the accurate synchrophasors. To improve such problem, an autoregressive (AR) model is applied. With the high resolution for the frequency estimation, the AR model can effectively detect the significant frequency components in a power signal. Since the AR model can be easily implemented with the recursive mechanism, the analysis structure of ADALINE can be used.

With the information of significant frequency components obtained in the first stage, the decomposition model of conventional ADALINE in the second stage can be remedied. In this way, the estimation of synchrophasors (including magnitudes and phase angles) would not be interfered with the power quality disturbances. To verify the performance of developed two-stage ADALINE synchrophasor

\footnotetext{
* Manuscript received May 14, 2013; revised July 28, 2013.

Corresponding author Tel.: +886-3-4227151 ext. 34526; E-mail address: hq7296@ yahoo.com.tw.
} 
estimator, the evaluation index of total vector error (TVE) and related testing requirements in IEEE Std. C37.118.1-2011 are applied [8]. In addition, several power system simulations and actual field measurements are performed to display the practicality and effectiveness of proposed synchrophasor estimator.

With the developed synchrophasor estimator, the benefits can be obtained as follows.

1. Using the AR model to estimate the time-varying frequencies, the resampling process of synchronization is not necessary.

2. The proposed two-stage ADALINE is not window-based technique, so no corrections or windowing issues would be encountered.

3. The estimation results meet the compliance requirements in IEEE Std. C37.118.1-2011 and the performance of proposed synchrophasor estimator would not be interfered with the power quality disturbances.

\section{Solution Mechanism of Two-Stage ADALINE}

To perform the synchrophasor estimation from the power signal contaminated with power quality disturbances, the signal under analysis can be generally represented in the discrete-time form, $y(n)$, of finite length $N$ sampled at the time interval $\Delta t$ by $H$ sinusoidal components as

$$
y(n)=\sum_{h=1}^{H} A_{h}(n) \cos \left(n \omega_{h}(n) \Delta t+\phi_{h}(n)\right)
$$

where $A_{h}(n)$ is the time-varying amplitude, $\phi_{h}(n)$ is the time-varying phase angle, $\omega_{h}(n)=2 \pi h f_{1}(n)$ is the time-varying harmonic radian frequency, and $f_{1}(n)$ is the time-varying power system frequency. After obtaining the amplitude and phase angles, the $h$ th harmonic phasor $Y_{h}(n)$ can be represented as

$$
Y_{h}(n)=\left(A_{h}(n) / \sqrt{2}\right) e^{j \phi_{h}(n)}
$$

In order to evaluate the accuracy of phasor estimation, the TVE between the measured (MEAS) and expected (IDEAL) phasors at a given instant of time $n$ is introduced in IEEE Std. C37.118.1-2011 [8], as given in (3).

$$
\operatorname{TVE}_{h}(n)=100 \% \cdot \frac{\left|Y_{h \_ \text {MEAS }}(n)-Y_{h \_ \text {IDEAL }}(n)\right|}{\left|Y_{h_{-} \text {DEAL }}(n)\right|}
$$

In the traditional ADALINE method, the signal model in (1) is further decomposed as

$$
\begin{aligned}
y(n) & =\sum_{h=1}^{H}\left(A_{h}(n) \cos \phi_{h}(n) \cos 2 \pi h f_{1}(n) n \Delta t-A_{h}(n) \sin \phi_{h}(n) \sin 2 \pi h f_{1}(n) n \Delta t\right) \\
& =\sum_{h=1}^{H}\left(w_{2 h-1}(n) \cos 2 \pi h f_{1}(n) n \Delta t-w_{2 h}(n) \sin 2 \pi h f_{1}(n) n \Delta t\right)
\end{aligned}
$$

where $w_{2 h-1}(n)$ and $w_{2 h}(n)$ are the weights of the ADALINE, which can be obtained with the gradient decent approach in $(5)$, where $W(n)=\left[w_{11}(n), w_{12}(n), \ldots, w_{2 H-1}(n), w_{2 H}(n)\right]^{\mathrm{T}}, x(n)=\left[\cos 2 \pi f_{1}(n) n \Delta t\right.$, $\left.\sin 2 \pi f_{1}(n) n \Delta t, \ldots, \cos 2 \pi H f_{1}(n) n \Delta t, \sin 2 \pi H f_{1}(n) n \Delta t\right]^{\mathrm{T}}, \alpha$ is the learning rate of ADALINE, and $e(n)$ is the estimation error between the actual and measured signals at time instant $n$.

$$
W(n+1)=W(n)+\Delta W(n)=W(n)+\frac{\alpha e(n) x(n)}{\lambda+x^{T}(n) x(n)}
$$

Then, the amplitude and phase angle of $h$ th harmonic phasor can be obtained with (6) and (7).

$$
A_{h}=\sqrt{w_{2 h-1}^{2}+w_{2 h}^{2}}
$$




$$
\phi_{h}=\tan ^{-1}\left(\frac{w_{2 h}}{w_{2 h-1}}\right)
$$

However, the analysis in the above relationship of ADALINE is based on the assumption that the power system frequency is fixed to its nominal value. Once the power system frequency deviation and interharmonics are present, the estimation errors for the synchrophasors would be increased. To deal with this problem, the two-stage ADALINE structure proposed by authors in [7] can be applied, as shown in Fig. 1. According to relationship of autoregressive (AR) model, the sampled signal can be written with linear prediction as [7]

$$
a_{0} y(n)=-\sum_{m=1}^{M} a_{m} y(n-m) \Rightarrow \sum_{m=0}^{M} a_{m} y(n-m)=0
$$

where coefficients $a_{m}$ s are real numbers and $M$ is the estimation order of AR model. If it is possible to minimize the estimation error for each $n$, the actual data sample and the estimated one will be identical. From [7], it is known that the coefficients $a_{m}$ s are related to the transfer function of AR model, as expressed in (9).

$$
a_{0} z^{M}+a_{1} z^{M-1}+\mathrm{L}+a_{M-1} z+a_{M}=0
$$

where $z_{k}=e^{j 2 \pi f_{h} \Delta t} \mid k=1,2, \mathrm{~K}, M$, and $f_{h}$ is the harmonic frequency, which is not necessary to be the integral multiple of power system frequency. Therefore, the AR model in (8) can be easily implemented with ADALINE. Once the correct frequencies are obtained, the harmonic phasors can be accurately calculated in the second stage of ADALINE.

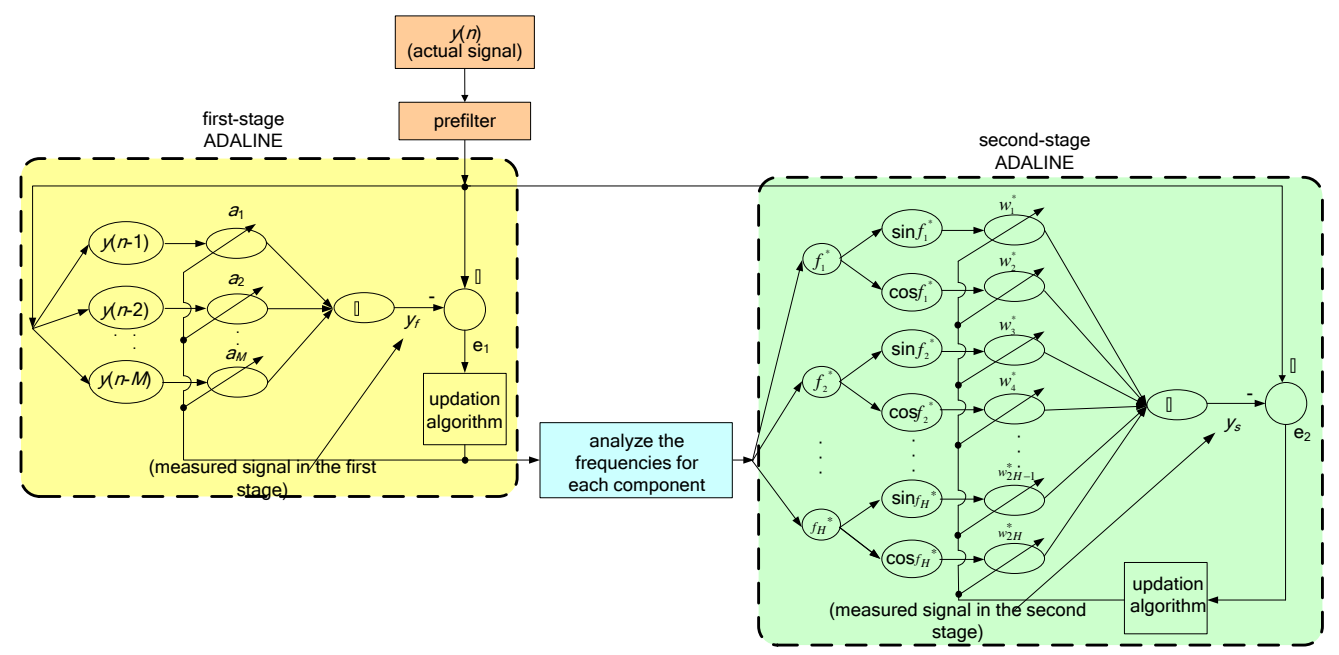

Fig. 1. Solution structure of two-stage ADALINE.

\section{Performance Evaluation for Proposed Measurement Mechanism}

To verify the performance of proposed two-stage ADALNE for the synchrophasor measurement, several compliance tests listed in IEEE Std. C37.118.1-2011 are performed [8], as shown in Table 1. The test results with conventional and proposed ADALINE methods are displayed in Fig. 2. From the comparisons, it is found that the two-stage ADALINE method meets the accuracy requirements of synchrophasor measurement. Due to the assumption of fixed nominal power system frequency in the analysis model of conventional ADALINE, the estimation related to variations of frequencies in conditions $\mathrm{C} 1, \mathrm{C} 4$, and $\mathrm{C} 5$ would result in larger estimation errors. 
Table 1. Compliance tests for synchrophasor in IEEE Std. C37.118.1-2011 [8]

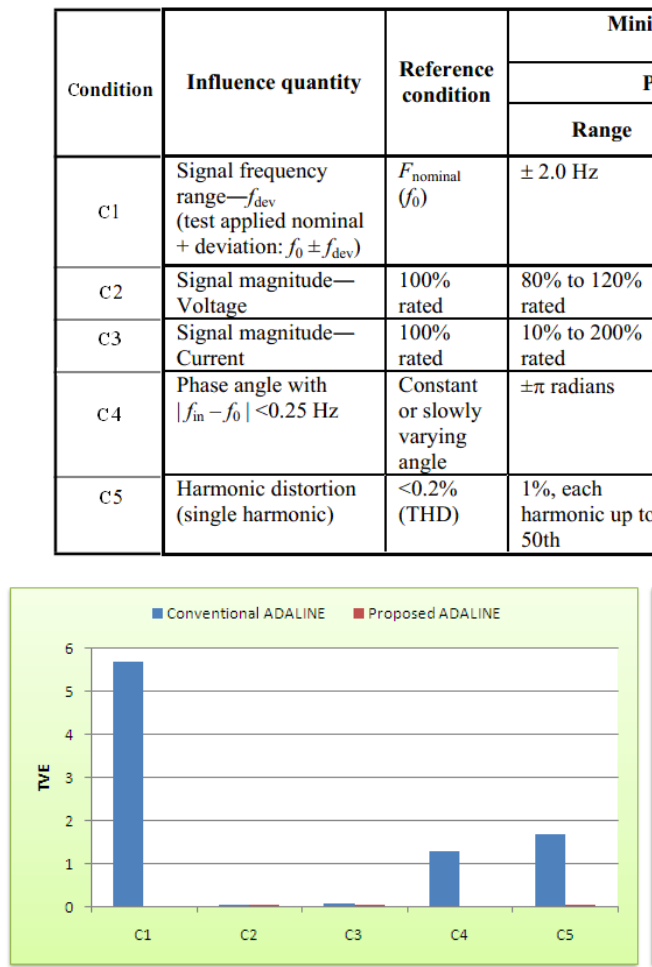

(a)

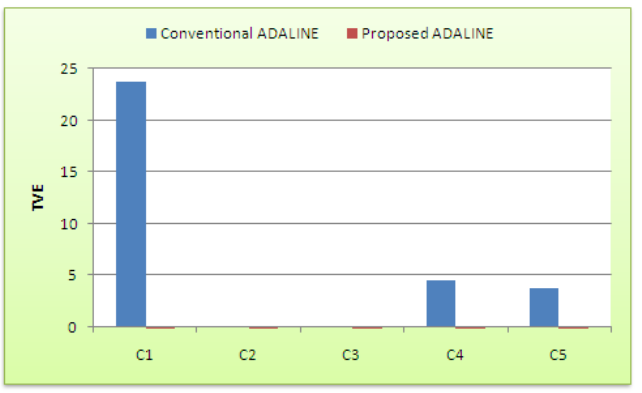

(b)

Fig. 2. Maximum TVE of compliance tests for (a) P class and (b) M class in IEEE Std. C37.118.1-2011.

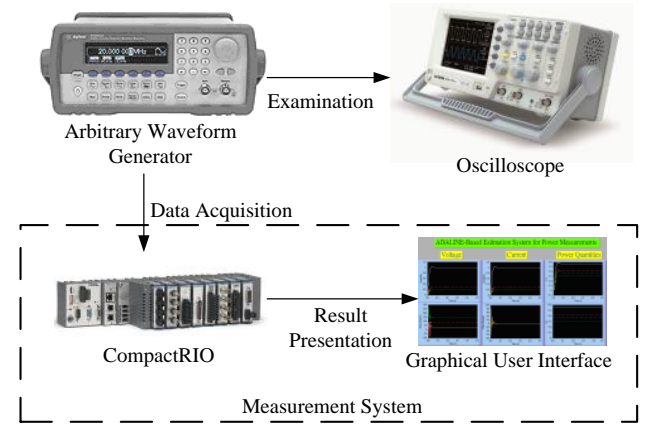

Fig. 3. Experimental setup for harmonic phasor estimation.

To realize the effectiveness of harmonic phasor estimation, a measurement system based on LabVIEW and National Instruments (NI) CompactRIO is established, as depicted in Fig. 3. A power signal in (10) is synthesized by the arbitrary waveform generator and extracted through the analog-to-digital converter on CompactRIO with sampling rate of $7680 \mathrm{~Hz}$, which is the integer multiple of nominal power system frequency. To examine the robustness of phasor estimation to the power quality disturbances, the power system frequency is set to be deviated from $60 \mathrm{~Hz}$ to $59.7 \mathrm{~Hz}$, and 3rd and 5th harmonics are included.

$$
v(t)=\sin \left(2 \pi \times 59.7+30^{\circ}\right)+0.3 \sin \left(3 \times 2 \pi \times 59.7+90^{\circ}\right)+0.2 \sin \left(5 \times 2 \pi \times 59.7+150^{\circ}\right)
$$

From the amplitude measurement in Fig. 4, it is found that there are slight influences on the estimated amplitudes with the conventional ADALINE. This is because the power system frequency deviation only introduces very small disturbances for weights in the calculation of amplitude in (6). In the proposed two- 
stage ADALINE, the effect of power system frequency deviation can be perfectly prevented. Due to the fixed nominal power system frequency used in the analysis model of conventional ADALINE, the estimated phase angles would rotate in the complex plane as illustrated in IEEE Std. C37.118.1-2011, as displayed in Fig. 5 (a), even though the phase angles in (10) are fixed values. In this way, the difficulty of phasor identification would lead to inconvenience in the related power system applications. On the contrary, the phase angles can be accurately detected and maintained in the fixed state by using the proposed solution mechanism, as represented in Fig. 5 (b).

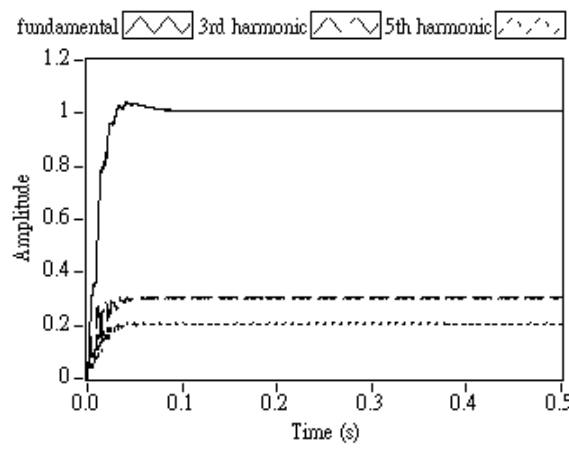

(a)

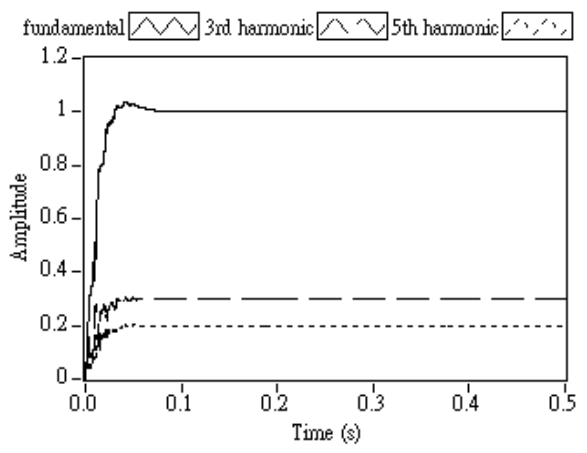

(b)

Fig. 4. The estimated amplitudes with (a) conventional ADALINE and (b) proposed two-stage ADALINE.

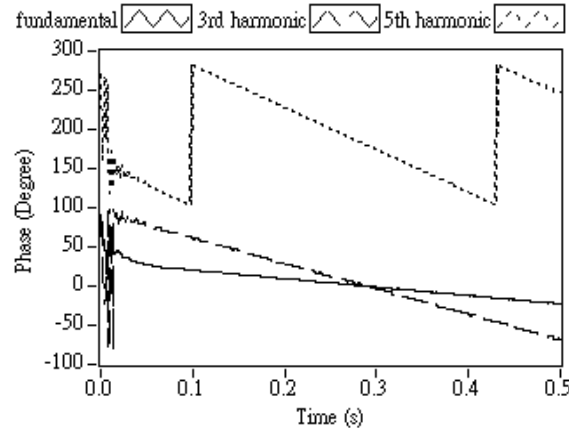

(a)

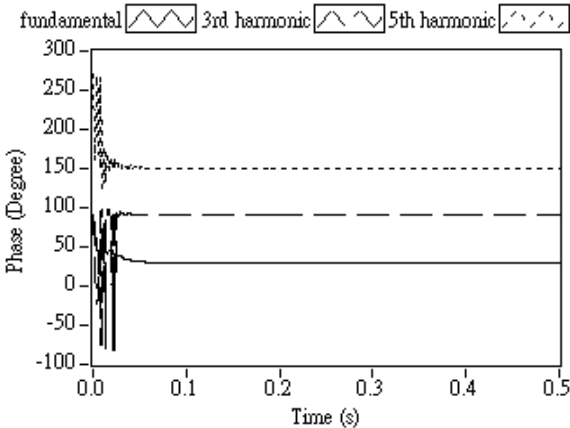

(b)

Fig. 5. The estimated phase angles with (a) conventional ADALINE and (b) proposed two-stage ADALINE.

\section{Conclusions}

In this paper, the two-stage ADALINE has been applied for the measurement of harmonic synchrophasors. With the high frequency resolution of AR model, the accurate frequency information for each harmonic component can be obtained without the traditional synchronization mechanism in IEC Std. 61000-4-7 [9]. After feeding the extracted frequency information into the second stage of ADALINE, the original structure could perform the synchrophasor measurement accurately. Different from the traditional methods illustrated in IEEE Std. C37.118.1-2011, the estimated harmonic phasor with proposed two-stage ADALINE would not rotate in the complex plane when the power system frequency deviates from the nominal value. This is because variant phase angles would cause inconvenience for the identification of direction of power flow, operation of protective relays, and interconnection of power grids. Therefore, the proposed synchrophasor measurement mechanism would provide accurate estimation results for the widearea monitoring.

\section{Acknowledgements}

The authors would like to acknowledge the financial support by the National Science Council of Taiwan, Republic of China, under Grants NSC 102-3113-P-194-002 for this work. 


\section{References}

[1] Yazdani D, Bakhshai A, Joos G, Mojiri M. A real-time extraction of harmonic and reactive current in a nonlinear load for grid-connected converters. IEEE Trans. on Industrial Electronics, 2009; 56(6):2185-2189.

[2] Fedele G, Picardi C, Sgro D. A power electrical signal tracking strategy based on the modulating functions method. IEEE Trans. on Industrial Electronics, 2009; 56(10):4079-4087.

[3] Yang S, Lei Q, Peng FZ, Qian Z. A robust control scheme for grid-connected voltage-source inverters. IEEE Trans. on Industrial Electronics, 2011; 58(1): 202-212.

[4] Marei MI, El-Saadany EF, Salama MMA. Envelope tracking techniques for flicker mitigation and voltage regulation. IEEE Trans. on Power Delivery, 2004; 19(4):1854-1861.

[5] Singh B, Verma V, Solanki J. Neural network-based selective compensation of current quality problems in distribution system. IEEE Trans. on Industrial Electronics, 2007; 54(1):53-60.

[6] Abdeslam DO, Wira P, Merckle J, Flieller D, Chapuis YA. A unified artificial neural network architecture for active power filters. IEEE Trans. on Industrial Electronics, 2007; 54(1):61-76.

[7] Chang GW, Chen CI, Liang QW. A two-stage ADALINE for harmonics and interharmonics measurement. IEEE Trans. on Industrial Electronics, 2009; 56(6):2220-2228.

[8] IEEE Standard for Synchrophasor Measurements for Power Systems. IEEE Std. C37.118.1 (2011).

[9] Testing and measurement techniques - General guide on harmonics and interharmonics measurements and instrumentation, for power supply systems and equipment connected thereto. IEC Std. 61000-4-7 (2009). 